Abanico Veterinario. Enero-Diciembre 2020; 10:1-7 http://dx.doi.org/10.21929/abavet2020.33 Artículo Original. Recibido: 05/01/2020. Aceptado: 03/09/2020. Publicado: 30/11/2020. Clave:2020-27.

\title{
Sincronización de estros en ovejas Pelibuey utilizando CIDR y diferentes dosis de eCG
}

\author{
Estrous synchronization of Pelibuey ewes, using CIDR and different doses of eCG
}

\section{Espinosa-Martínez Mario*1 ID , Montiel-Olguín Luis ${ }^{1 \mathrm{ID}}$, Villaseñor-González Fernando² ID $^{2}$, Jiménez-Severiano Héctor ${ }^{1}$ ID}

${ }^{1}$ Centro Nacional de Investigación Disciplinaria en Fisiología y Mejoramiento Animal-Instituto Nacional de Investigaciones Forestales, Agrícolas y Pecuarias México. ${ }^{2}$ Campo Experimental Centro Altos de JaliscoInstituto Nacional de Investigaciones Forestales, Agrícolas y Pecuarias. México. *Autor responsable y de correspondencia: Espinosa-Martínez Mario. Km 1 Carr. Ajuchitlán-Colón, Ajuchitlán, Colón, Querétaro, México. CP 76280. marespinosa71@yahoo.com, montiel.luis@inifap.gob.mx, villasenor.fernando@inifap.gob.mx, jimenez.hector@inifap.gob.mx

\section{Resumen}

En la raza ovina Pelibuey, no está establecida completamente la dosis de gonadotropina coriónica equina (eCG) a emplear en protocolos de sincronización de estros. El objetivo del estudio fue analizar el efecto de dos diferentes dosis de eCG, incluidas en protocolos de sincronización de estros basados en progestágenos, sobre la respuesta reproductiva de ovejas Pelibuey. Para ello, 28 y 24 ovejas durante dos años consecutivos, fueron sometidas a un protocolo de sincronización; el primer grupo (BAJA) recibió un CIDR durante siete días y a su retiro se administró $0.125 \mathrm{mg}$ de Cloprostenol y eCG a una dosis i.m. de $300 \mathrm{U}$. El segundo grupo (ALTA) recibió el mismo protocolo con una modificación, el empleo de una dosis de $400 \mathrm{U}$ de eCG. Para evaluar las variables obtenidas, se realizó la prueba exacta de Fisher (datos en porcentaje) 0 análisis de varianza. No existieron diferencias $(P>0.05)$ entre grupos para el porcentaje de ovejas en estro (92.31\%), la tasa de concepción total $(82.69 \%)$ y de las ovejas con estro sincronizado $(87.50 \%)$. Adicionalmente, el inicio del estro $(47.75 \pm 1.43 \mathrm{~h})$ y la prolificidad ( 1.86 crías/parto) fue similar $(P>0.05)$ entre grupos. En conclusión, los protocolos de sincronización de estros para ovejas Pelibuey que utilizan CIDR por siete días, pueden emplear eCG a una dosis de al menos $300 \mathrm{U}$, sin afectar sus indicadores reproductivos asociados.

Palabras clave: ovinos, gonadotropina coriónica equina, tasa de concepción.

\begin{abstract}
In the Pelibuey breed, the dose of equine chorionic gonadotropin (eCG) used in the estrous synchronization is not fully established. The objective of this study was to analyze the effect of two different doses of eCG included in progestagens-based estrous synchronization protocols, on the reproductive response of Pelibuey ewes. For this reason, 28 and 24 ewes during two consecutive years were submitted to a synchronization protocol. The first group (LOW) considered the use of a CIDR for a 7-day period, followed by an i.m. administration of $0.125 \mathrm{mg}$ of Cloprostenol and a dose of $300 \mathrm{U}$ of eCG. The second group (HIGH) received the same protocol with a modification, the use of a $400 \mathrm{U}$ of eCG. To evaluate the response variables, Fisher exact test and analysis of variance, were considered. There was no difference $(P>0.05)$ between groups for the percentage of estrous ewes (92.31\%), total conception rate (82.69\%) and the conception rate of ewes with synchronized estrous (87.5\%). Additionally, the estrous beginning $(47.75 \pm 1.43$ h) and prolificacy (1.86 lambs/partum) was similar between groups. In conclusion, the estrous synchronization protocols for Pelibuey ewes using CIDR for a 7-day period, can use eCG in a dose of 300 $U$ without effects on their associated reproductive performance.
\end{abstract}

Keywords: ovine, equine chorionic gonadotropin, conception rate. 


\section{INTRODUCCIÓN}

En diferentes especies domésticas, desde hace varias décadas se emplea como práctica rutinaria en algunas unidades de producción, una tecnología reproductiva que es la sincronización de estros. Su uso en la especie ovina permite mejorar la eficiencia reproductiva; aunque factores como la amplia variedad de los protocolos de sincronización (Hashemi et al., 2006) y los productos hormonales empleados entre los que están los progestágenos (Fleish et al., 2012; Hashemi et al., 2006), pueden afectar la respuesta reproductiva a estos protocolos y con ello aumentar los costos de producción. Una hormona incluida de manera complementaria en los protocolos es la gonadotropina coriónica equina (eCG), favoreciendo la tasa de concepción y la tasa ovulatoria; aunque un riesgo asociado con el uso de esta hormona es el aumento de la tasa ovulatoria, lo que lleva a tener nacimientos múltiples y la posibilidad de tener crías de bajo peso; lo que compromete su sobrevivencia (Lozano-González et al., 2012). Diversos estudios consideran el uso de eCG, en dosis que van desde 0 hasta $500 \mathrm{U}$ (Arroyo-Ledezma et al., 2013; Fleish et al., 2012; Garoussi et al., 2019; lida et al., 2004); sin embargo, prácticamente no existen estudios que justifiquen el uso de estas diferentes dosis en la oveja Pelibuey; una raza considerada de baja estacionalidad (Arroyo et al., 2007); por lo que el uso de las dosis altas podrían no estar justificadas. Una reducción en la dosis empleada permitiría reducir el costo del protocolo de sincronización, continuar el tránsito hacia protocolos con menor uso de hormonas y disminuir los riesgos asociados por la presencia de nacimientos múltiples.

Por lo anterior, el objetivo del presente trabajo fue analizar el efecto de dos diferentes dosis de eCG, incluidas en protocolos de sincronización de estros, basados en progestágenos sobre la respuesta reproductiva de ovejas Pelibuey.

\section{MATERIAL Y MÉTODOS}

El estudio se llevó a cabo en los meses de febrero y marzo (invierno tardío) de dos años consecutivos, en el municipio de Colón, en el estado de Querétaro, México (2042' Latitud Norte y $100^{\circ} 01^{\prime}$ Longitud Oeste). En esta región prevalece un clima templado semiseco, con una temperatura promedio anual de 17.4으. Ovejas Pelibuey $(n=28$ en el año 1 y 24 en el año 2) fueron utilizadas, las cuales al inicio del estudio contaban con un peso promedio de $50.1 \pm 1.36 \mathrm{~kg}$ y una condición corporal promedio de $3.41 \pm 0.07$, medido en una escala de 1 a 5 (Romero, 2015). Las ovejas fueron asignadas al azar a dos protocolos de sincronización de estros; el primer grupo (BAJA) recibió la inserción de un dispositivo intravaginal, conteniendo progesterona (CIDR) el día 0; mismo que fue retirado al día 6, en conjunto con la aplicación de una dosis i.m. de $300 \mathrm{U}$ de eCG y una dosis de 0.125 $\mathrm{mg}$ de un análogo de la prostaglandina F2 alfa (Cloprostenol). El segundo grupo (ALTA), recibió el CIDR al día 0, retirándose al día 6, en conjunto con la aplicación de una dosis mayor de eCG (400 U) y una dosis de $0.125 \mathrm{mg}$ de Cloprostenol.

A las $24 \mathrm{~h}$ de haber retirado los dispositivos, las ovejas fueron sometidas a detección de estros. La detección se realizó dos veces por día (09:00 h y 18:00 h), empleando para 
ello un semental de la misma raza, hasta cumplir $72 \mathrm{~h}$. El servicio se realizó por monta natural, procurando una proporción macho:hembra no mayor a 1:10.

Posteriormente, las ovejas fueron sometidas a diagnósticos de gestación 35 días después de su servicio, para determinar la tasa de concepción; para ello, se utilizó un ultrasonido marca Aloka, con un transductor transrectal de $5 \mathrm{MHz}$ (Mod. SSD500). Al nacimiento, se registró el número de crías nacidas para cada una de las hembras, para determinar su prolificidad.

Para el análisis estadístico, se realizó la prueba exacta de Fisher, para establecer posibles diferencias en los porcentajes de ovejas en estro y de ovejas gestantes. Adicionalmente, se realizaron análisis de varianza para determinar diferencias entre grupos para las variables peso vivo, condición corporal, el inicio del estro y la prolificidad; considerando un diseño de bloques (año) completos aleatorizados. Previamente, los datos de algunas variables fueron transformados mediante su logaritmo natural; sin embargo, se muestran sus valores no transformados para facilitar su lectura.

El inicio del estro fue considerado tomando en cuenta el tiempo transcurrido entre el retiro del CIDR y la primera conducta estral franca (la oveja permitía su monta, sin moverse). Los datos fueron analizados mediante el paquete estadístico SAS (SAS Institute Inc., Cary, NC), considerando en todos los análisis un valor de $\mathrm{P}<0.05$, como significancia estadística (Chavalarias et al., 2016).

\section{RESULTADOS Y DISCUSIÓN}

En este estudio, la condición corporal puede afectar indicadores reproductivos (De La Isla et al., 2010) y el peso de las ovejas; al inicio del estudio fue similar $(P>0.05)$ entre los grupos de sincronización (cuadro 1), lo que permite asegurar que alguna posible diferencia entre los tratamientos estudiados no estaría asociada a estos indicadores, y que ambos grupos ingresaron al estudio en igualdad de condiciones. La condición corporal registrada está dentro de lo deseable para ovejas que entrarán a un periodo de servicio reproductivo. Sólo para esta variable, la inclusión del año como bloque resultó significativo $(\mathrm{P}<0.05)$.

Cuadro 1. Variables reproductivas promedio de ovejas Pelibuey sometidas a dos protocolos de sincronización de estros, empleando CIDR por siete días y una dosis baja o alta de eCG

\begin{tabular}{lccc}
\hline Variable & Baja & Alta & Total \\
\hline $\mathrm{n}$ & 27 & 25 & 52 \\
Peso vivo, kg & $49.75 \pm 1.78$ & $50.40 \pm 2.10$ & $50.06 \pm 1.36$ \\
Condición corporal & $3.43 \pm 0.10$ & $3.39 \pm 0.11$ & $3.41 \pm 0.07$ \\
Inicio del estro, $\mathrm{h}$ & $48.36 \pm 1.77$ & $47.09 \pm 2.33$ & $47.75 \pm 1.43$ \\
Prolificidad (corderos/parto) & $1.95 \pm 0.15$ & $1.76 \pm 0.12$ & $1.86 \pm 0.1$ \\
\hline
\end{tabular}


Aunque no se observaron diferencias $(P>0.05)$ entre los tratamientos para el porcentaje de estros (fig. 1), la respuesta alcanzada para este indicador fue buena, tomando en cuenta lo que se ha descrito para ovejas con protocolos de sincronización similares (Ali et al., 2009), y mejor que aquellos que han empleado esponjas intravaginales (Alavez et al., 2014). Por otro lado, un indicador quizá más importante, como lo es la tasa de concepción, tampoco fue afectada ( $P>0.05)$, por la dosis de eCG empleada.

Los resultados son ligeramente mayores a estudios con ovejas empleando esponjas (Garoussi et al., 2019). Esto permite afirmar que el uso de una dosis de sólo $300 \mathrm{U}$ de eCG puede ser tan eficiente para favorecer la expresión de estros y la concepción de las ovejas, como una dosis mayor (400 U). En los protocolos de sincronización de estros utilizados, la eCG es la de segundo mayor costo, por lo que una disminución en su dosis puede representar un ahorro considerable. Sin embargo, aún queda por establecer si esta dosis puede ser aún menor para la raza empleada.

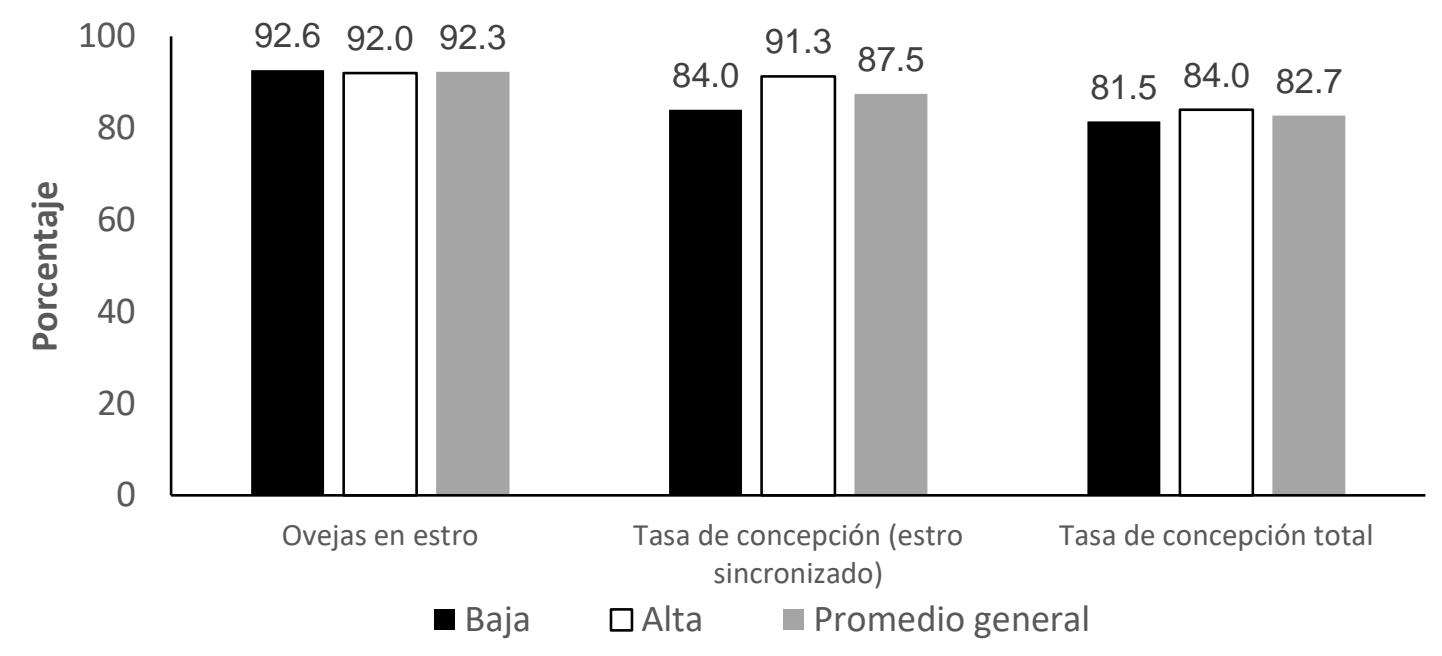

Figura 1. Porcentaje de ovejas en estro y tasa de concepción de ovejas con protocolo de sincronización de estros que incluyo eCG a dosis baja o alta

El porcentaje de ovejas en estro presentó resultados similares entre ambos grupos desde las 24 y hasta las $72 \mathrm{~h}$ (figura 2). Los resultados obtenidos permiten sugerir que el control de ovejas en estro se realice a partir de las 33 horas, debido a que a las $24 \mathrm{~h}$, ninguna de las ovejas mostró estro. 


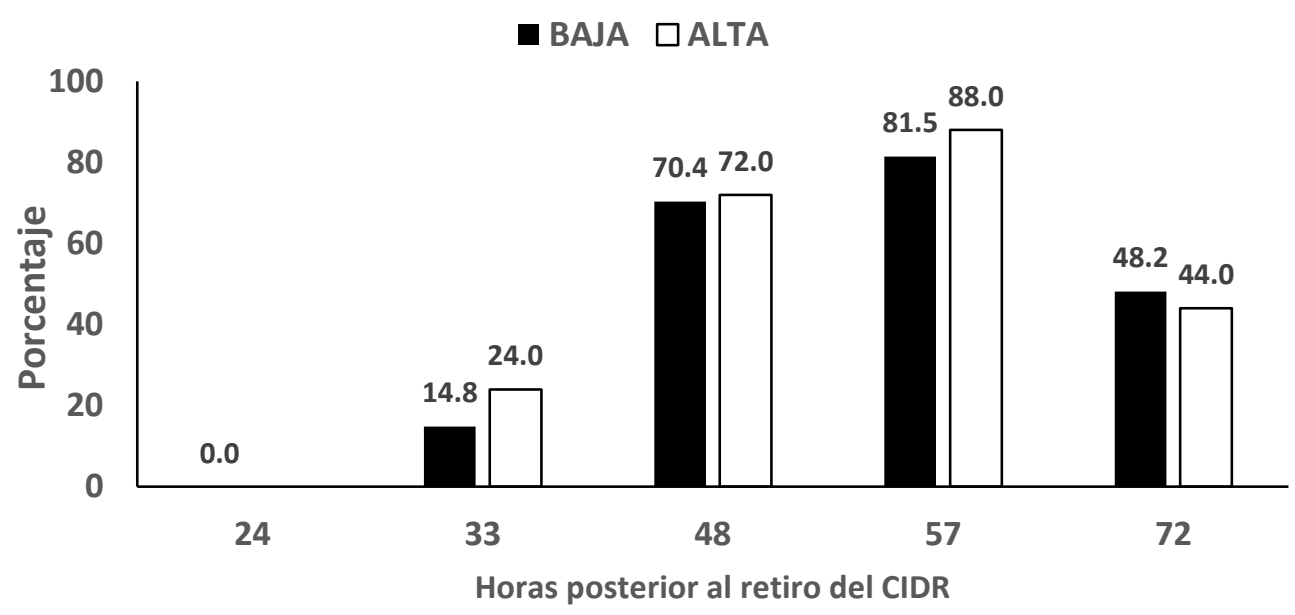

Figura 2. Porcentaje de ovejas en estro, que fueron sincronizadas con CIDR y dosis baja o alta de eCG

El inicio del estro se presentó a un promedio similar $(P>0.05)$, entre grupos de sincronización (cuadro 1), con promedios que fueron mayores a lo observado en otros estudios empleando CIDR (Ali et al., 2009; Arroyo et al., 2013), o esponjas intravaginales (Alavez et al., 2014). La discrepancia entre estudios podrían ser debidas a las razas; ya que este inicio del estro fue similar a lo observado también con ovejas de pelo, empleando esponjas intravaginales (Alavez et al., 2014). Desafortunadamente la diversidad de condiciones empleadas en estos estudios limita una comparación más precisa. La eCG puede aumentar la tasa ovulatoria (Lozano-González et al., 2012); sin embargo, aunque se observó una menor prolificidad con el uso de una dosis alta de eCG, ésta no fue estadísticamente diferente. Los valores de prolificidad son superiores a lo descrito para la raza Pelibuey por Galina et al. (1996) y Magaña-Monforte et al. (2013).

Es probable que la alta prolificidad asociada a esta raza permita ser más flexible en cuanto al uso de eCG, incluso para utilizar dosis aún menores a las empleadas en este estudio, sin afectar o impactar de manera evidente los indicadores reproductivos evaluados. Estudios en el futuro deberán comprobar lo anterior.

\section{CONCLUSIONES}

El uso de una dosis de $300 \mathrm{U}$ de gonadotropina coriónica equina, puede ser empleada en protocolos de sincronización de estros de ovejas Pelibuey, basados en el uso de dispositivos intravaginales de liberación controlada de progesterona. Esta dosis no modifica la respuesta reproductiva de las ovejas, en comparación al uso de dosis mayores de esta hormona. Estudios en el futuro deberán establecer si es posible emplear dosis aún menores para esta raza, además de considerar el costo de los protocolos empleados. 


\section{Agradecimientos}

Se agradece al Instituto Nacional de Investigaciones Forestales, Agrícolas y Pecuarias, por el apoyo brindado a través de la Unidad Experimental Ovina mediante el proyecto SIGI 166213934.

\section{LITERATURA CITADA}

ALAVEZ RA, Arroyo LJ, Montes PR, Zamora BR, Navarrete SLF, Magaña SH. 2014. Short communication: Estrus synchronization using progestogens or cloprostenol in tropical hair sheep. Tropical Animal Health and Production. 46(8): 1515-1518. https://doi.org/10.1007/s11250-014-0660-z

ALI A, Hayder M, Saifelnaser EOH. 2009. Ultrasonographic and endocrine evaluation of three regimes for oestrus and ovulation synchronization for sheep in the subtropics. Reproduction in Domestic Animals. 44: 873-878. https://doi.org/10.1111/j.14390531.2008.01102.x

ARROYO LJ, Gallegos-Sánchez J, Villa-Godoy A, Berruecos JM, Perera G, Valencia J. 2007. Reproductive activity of Pelibuey and Suffolk ewes at 19- north latitude. Animal Reproduction Science. 102: 24-30. https://doi.org/10.1016/j.anireprosci.2006.09.025

ARROYO-LEDEZMA J, De La Torre-Barrera J, Ávila-Serrano NY. 2013. Respuesta reproductiva de ovejas de pelo sincronizadas con progesterona o prostaglandinas. Agrociencia. 47:661-670. ISSN 1405-3195.

http://www.scielo.org.mx/pdf/agro/v47n7/v47n7a3.pdf

CHAVALARIAS D, Wallach JD, Ho TLA. 2016. Evolution of reporting P values in the biomedical literature, 1990-2015. Journal of the American Medical Association. 315: 11411148. https://doi.org/10.1001/jama.2016.1952

DE LA ISLA HG, Aké LJR, Ayala BA, González-Bulnes A. 2010. Efecto de la condición corporal y la época del año sobre el ciclo estral, estro, desarrollo folicular y tasa ovulatoria en ovejas Pelibuey mantenidas en condiciones de trópico. Veterinaria México. 41(3). ISSN-0301-5092. http://www.scielo.org.mx/pdf/vetmex/v41n3/v41n3a1.pdf

FLEISH A, Werne S, Heckendorn F, Hartnack S, Piechotta M, Bollwein H, Thun R, Janett F. 2012. Comparison of 6-day progestagen treatment with Chronogest $C R$ and Ezi-breed CIDR G intravaginal inserts for estrus synchronization in cyclic ewes. Small Ruminant Research. 107: 141-146. http://dx.doi.org/10.1016/j.smallrumres.2012.05.014

GALINA MA, Morales R, Silva E, López B. 1996. Reproductive performance of Pelibuey and Blackbelly sheep under tropical management systems in Mexico. Small Ruminant Research. 22: 31-37. https://doi.org/10.1016/0921-4488(95)00878-0

GAROUSSI MT, Mavadati O, Bahonar M, Ragh MJ. 2019. The effect of medroxyprogesterone acetate with or without eCG on conception rate of fat-tail ewes in out of breeding season. Tropical Animal Health and Production. https://doi.org/10.1007/s11250-019-02159-8 
HASHEMI M, Safdarian M, Kafi M. 2006. Estrous response to synchronization of estrus using different progesterone treatments outside the natural breeding season in ewes. Small Ruminant Research. 279-283. https://doi.org/10.1016/j.smallrumres.2005.07.051

IIDA K, Kobayashi N, Kohno H, Miyamoto A, Fukui Y. 2004. A comparative study of induction of estrus and ovulation by three different intravaginal devices in ewes during the non-breeding season. Journal of Reproduction and Development. 50(1):63-69. https://doi.org/10.1262/jrd.50.63

LOZANO-GONZÁLEZ JF, Uribe-Velásquez LF, Henry OJ. 2012. Control hormonal de la reproducción en hembras ovinas. Veterinaria Zootecnia. 6(2):134-147. ISSN 2011-5415 http://vetzootec.ucaldas.edu.co/downloads/v6n2a10.pdf

MAGAÑA-MONFORTE JG, Huchin-Cab M, Ake-López RJ, Segura-Correa JC. 2013. A field study of reproductive performance and productivity of Pelibuey ewes in Southeastern Mexico. Tropical Animal Health and Production. 45(8): 1771-1776. https://doi.org/17711776. 10.1007/s11250-013-0431-2

ROMERO O. 2015. Evaluación de la condición corporal y edad de los ovinos. Herramientas de manejo animal. Informativo. Instituto de Investigaciones Agropecuarias$\begin{array}{lllll}\text { Ministerio de Agricultura. Temuco } & \text { Chile. } & \text { No. }\end{array}$ http://biblioteca.inia.cl/medios/biblioteca/informativos/NR40188.pdf

SAS Institute. 2011. Statistical Analysis Software SAS/STAT. Base SAS 9.3. Procedures Guide Statistical Procedures. Cary, N.C., USA:SAS Institute Inc., ISBN: 978-1-60764896-3. https://support.sas.com/documentation/onlinedoc/base/procstat93m1.pdf 